\title{
Prostate Shape Modeling Based on Principal Geodesic Analysis Bootstrapping
}

\author{
Erik Dam ${ }^{1}$, P. Thomas Fletcher ${ }^{2}$, Stephen M. Pizer ${ }^{2}$, \\ Gregg Tracton ${ }^{3}$, and Julian Rosenman ${ }^{3}$ \\ 1 Image Analysis group, IT University of Copenhagen, and \\ Center for Clinical and Basic Research, erikdam@itu.dk \\ 2 Medical Image Display and Analysis Group, Uni. of North Carolina, Chapel Hill \\ 3 Dep. of Radiation Oncology, University of North Carolina, Chapel Hill
}

\begin{abstract}
The use of statistical shape models in medical image analysis is growing due to the ability to incorporate prior organ shape knowledge for tasks such as segmentation, registration, and classification.

Shape models are trained from collections of segmented organs. Though manual interaction during training can ensure correspondence, it also introduces bias and ruins reproducibility - automation is desirable.

We present a novel shape model construction method via a medial shape representation. The automatic method is based on an iterative bootstrap method that alternates between shape representation optimization and analysis of shape mean and variations.

The method is used to create a model from 46 segmented prostates with quantitatively and intutitively good results.
\end{abstract}

\section{Introduction}

Methods based on analysis of shape variation are widespread in medical imaging. These methods allow incorporation of statistical prior shape knowledge in tasks where image information alone is not enough to solve the task automatically. The classical example is the use of deformable models in segmentation.

Most statistical shape models consist of a mean shape with deformations that are constructed through statistical analysis of shapes from a training collection. Each shape is described partially by the chosen shape representation, and analysis of the parameters for the representation gives the mean and variations.

The best known is the Active Shape Model (ASM) [1] where the shapes are represented by a point distribution model (PDM) with given point-wise correspondence. The mean model is simply the mean of each point after Procrustes alignment. Principal component analysis (PCA) provides the variations.

This work pursues the medial shape representation $m$-rep [3]. The m-rep represents shape by means of the sheet of sampled medial atoms. This parameter space is not Euclidean but consists of a combination of position, scaling, and orientation parameters. Standard PCA is therefore not applicable. However, the analogue that applies to shape representations that form Lie groups is the Principal Geodesic Analysis (PGA) 4,5]. 
A key step in constructing shape models is the representation of the training shapes. This must define correspondence across the population. For a PDM the simplest method is manual selection of the boundary points by a medical expert. In $2 \mathrm{D}$, and especially $3 \mathrm{D}$, this is a time-consuming and non-reproducible. However, this is automated by Davies [6] by first generating boundary points from a spherical harmonics representation and then optimizing the boundary points and their correspondence in a Minimum Description Length (MDL) approach.

This work presents an essentially automatic shape modeling method. The essense is an automatic bootstrap process that iteratively fits the shape model to training shapes and then derives the PGA mean and modes of deformation. Through the bootstrap iterations, the PGA mean and variations are optimized to allow automatic fitting of all shapes in the training collection. The main difference compared to [6] is that the MDL approach starts the optimization process from representations with good training shape fit and poor correspondence. The MDL process then protects the shape fits while optimizing the correspondence. The PGA bootstrap starts from a generative model with explicit correspondence but with poor fit to the individual training shapes. The bootstrap process then keeps the correspondence while optimizing the fit to the training shapes.

Another method generates an m-rep mean model from training shapes through a spherical harmonics representation that is transformed to Voronoi skeletons [7. Our approach provides modes of variation as well and is cleaner since the m-rep is the only representation in play. A similar bootstrapping approach that uses an atlas/registration methodology instead is presented in [8].

We evaluate the presented PGA bootstrap for construction of a prostate shape model. The training collection consists of 46 cases where the prostates were segmented in the course of prostate cancer external-beam radiation treatment. Especially in CT scans with slice thickness $2 \mathrm{~mm}$ or larger, the boundaries of the prostate have low contrast — therefore, prior knowledge in a statistical shape model is essential to making automatic segmentation possible.

The contributions of this work are threefold: a) The PGA bootstrap method that allows essentially automatic generation of a shape model with mean and main modes of variation, $b$ ) Introduction of the necessary geometric regularization term for the m-rep, and $c$ ) The resulting prostate model that will be central in segmentation and analysis of prostates in radiation treatment planning.

\section{The UNC Pelvis Collection}

The slice-based segmentation programs, MASK [9] and anastruct_editor, from the PLan-UNC suite of radiotherapy treatment tools developed at UNC-CH Radiation Oncology, were used to manually produce binary segmentations. Prostatic fat is included in the prostate's shape, as is seen in clinical practise, both because of the difficulty of finding the border between these and the prostate and the chance that these will contain significant counts of cancer cells. Seminal vesicles are excluded from the prostate. 

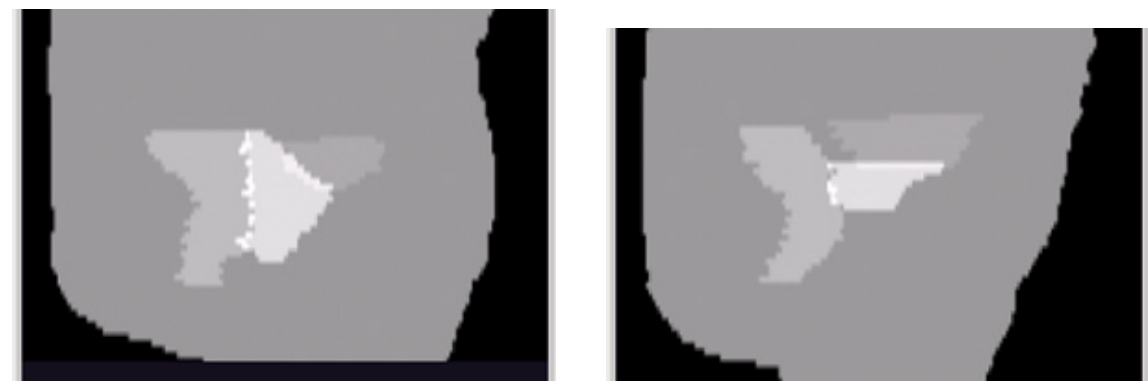

Fig. 1. Sagittal slices of the manual segmentations of rectum, prostate (brightest), and bladder (darkest) from two cases in the UNC pelvis collection. Notice the large variation in the shape of the segmented prostates. The volume varies from $12 \mathrm{~cm}^{3}$ to $144 \mathrm{~cm}^{3}$.

The ungated CT scans are acquired from non-immobilized supine patients at UNC Healthcare (Chapel Hill, NC, USA) and Western Wake Radiology (Cary, NC, USA) on Siemens Somotom $4+$ scanners without contrast agents.

Retrospective patient images are selected from the archives based on technical criteria, such as adequate image quality and anatomical coverage (the entire bladder down through the prostate apex), as well as shape and anatomical considerations such as very large bladders, prosthetic hips, or surgical procedures proximal to the prostate, yielding "normal cancerous" prostates.

The collection has 46 sets with manual segmentations for prostate, bladder, and rectum (see figure 1). All cases are diagnosed with prostate cancer so the resulting shape model will not necessarily model prostates in general. For instance, an increase of the size of the prostate is common for prostate cancer patients. Since the shape model is to be used for analysis of patients diagnosed with prostate cancer, this bias towards cancerous prostates is desirable.

\section{Medial Shape Representation: M-rep}

We use a medial shape representation, m-rep, and here briefly review the geometry and the framework for image segmentation [1011] and introduce a novel regularization term essential for the bootstrap framework.

The m-rep is based on the medial axis of Blum [12. In this framework, a 3D geometric object is represented as a set of connected continuous medial sheets, which are formed by the centers of all spheres that are interior to the object and tangent to the object's boundary at two or more points. Here we focus on 3D objects that can be represented by a single medial sheet.

We sample the medial sheet $\mathcal{M}$ over a spatially regular lattice of medial atoms defined as a 4-tuple $\mathbf{m}=\{\mathbf{x}, r, \mathbf{F}, \theta\}$, consisting of: $\mathbf{x} \in \mathbb{R}^{3}$ and $r \in \mathbb{R}^{+}$, the center and radius of the sphere, $\mathbf{F} \in \mathbf{S O}(3)$ an orthonormal local frame parameterized by $\left(\mathbf{b}, \mathbf{b}^{\perp}, \mathbf{n}\right)$, where $\mathbf{n}$ is the normal to the medial manifold, $\mathbf{b}$ is the direction in the tangent plane of the fastest narrowing of the implied boundary sections, 
and $\theta \in[0, \pi)$ the object angle determining the angulation of the two implied opposing boundary points to the local frame. Given an m-rep figure, we fit a smooth boundary surface to the model. We use a subdivision surface method [13] that interpolates the boundary positions and normals implied by each atom.

\subsection{Segmentation Using m-reps}

Following the deformable models paradigm, an m-rep model $\mathbf{M}$ is deformed into an image $I$ by optimizing an objective function:

$$
F(\mathbf{M}, I)=L(\mathbf{M}, I)+\alpha G(\mathbf{M})+R(\mathbf{M})
$$

The function $L$, the image match, measures how well the model matches the image information, while $G$, the geometric typicality, gives a prior on the possible variation of the geometry of the model weighted by $\alpha \geq 0$. The last term $R$, the geometric regularization, is a novel addition to the m-rep framework.

This objective function is optimized in a multiscale fashion. That is, it is optimized over a sequence of transformations that are successively finer in scale. Here we will only be concerned with the figural level and the medial atom level. At the figural level the transformation we use is a similarity transformation plus an elongation of the entire figure. At the atom level each medial atom is independently transformed by a similarity plus a rotation of the object angle.

M-rep models are fit to binary segmentation images of the prostates. These binary images are blurred slightly to smooth the objective function, which is optimized with a conjugate gradient method. The image match term of the objective function, $L$, is computed as a correlation with a Gaussian derivative kernel in the normal direction to the object boundary:

$$
L(\mathbf{M}, I)=\int_{\mathcal{B}(\mathbf{M})} \int_{-\epsilon}^{\epsilon} \partial_{t} G(t) I(\mathbf{s}+(t / r) \mathbf{n}) d t d \mathbf{s}
$$

where $\mathbf{s}$ is a parameterization of the boundary $\mathcal{B}(\mathbf{M}), \partial_{t} G$ is the Gaussian derivative kernel, $r$ is the radius function, and $\mathbf{n}$ is the boundary normal.

The geometric typicality term, $G$, is defined as the change in the boundary from the previous level of scale (where $\mathbf{s}_{0}$ is the boundary position at that level):

$$
G(\mathbf{M})=-\int_{\mathcal{B}(\mathbf{M})} \frac{\left\|\mathbf{s}-\mathbf{s}_{0}\right\|^{2}}{r^{2}} d \mathbf{s}
$$

The geometric regularization term, $R$, essentially corresponds to the curvature and neighbor distance terms in the active contour model and is added during the bootstrap iterations that are introduced in section [5 at the atom scale level in order to keep the model nice. For a medial atom $\mathbf{m}$ with neighbor atoms $\mathbf{m}_{\mathbf{i}}$ :

$$
\begin{aligned}
R(\mathbf{m})=\sum_{i=1}^{8} & \gamma_{\text {dist }} \operatorname{rd}\left[d\left(\mathbf{m}, \mathbf{m}_{\mathbf{i}}\right), \overline{d(\cdot, \cdot)}\right]^{2}+\gamma_{\text {implode }} \frac{\operatorname{diam}^{2}}{(N+1)^{2} d\left(\mathbf{m}, \mathbf{m}_{\mathbf{i}}\right)^{2}}+ \\
& \gamma_{\text {grid }} \text { angle }\left(\mathbf{m}-\mathbf{m}_{\mathbf{i}}, \mathbf{m}-\mathbf{m}_{-\mathbf{i}}\right)^{2}+\gamma_{\text {swirl }} \text { angle }\left(\mathbf{b}, \mathbf{b}_{\mathbf{i d e a l}}\right)^{2}
\end{aligned}
$$

Here $d(\cdot, \cdot)$ is the distance between atom centers (weighted by $1 / \sqrt{2}$ for diagonal neighbors), $\overline{d(\cdot, \cdot)}$ is the mean distance for the model, and $r d$ is the relative difference. Furthermore, diam is the model diameter, $N$ is the number of atoms, $\mathbf{m}_{-\mathbf{i}}$ is the neighbor opposing $\mathbf{m}_{\mathbf{i}}$ on the other side of $\mathbf{m}$, and $\mathbf{b}_{\text {ideal }}$ is the $\mathbf{b}$ vector as in an ideally oriented atom at that position in the lattice. 


\section{Principal Geodesic Analysis}

Principal geodesic analysis (PGA) [5] is a generalization of principal component analysis (PCA) to curved manifolds. We briefly review the results here.

As shown in [5], the set of all medial atoms forms a Lie group $M=\mathbb{R}^{3} \times \mathbb{R}^{+} \times$ $\mathbf{S O}(3) \times \mathbf{S O}(2)$, which we call the medial group. Likewise, the set of all m-rep models containing $n$ medial atoms forms a Lie group $M^{n}$, i.e., the direct product of $n$ copies of $M$. This allows the defintion of the exponential and logarithmic maps, $\exp (\cdot)$ and $\log (\cdot)$, that defines the geodesics of the medial group.

\subsection{M-rep Means and PGA}

The Riemannian distance between m-rep models $\mathbf{M}_{1}, \mathbf{M}_{2} \in M^{n}$ is given by $d\left(\mathbf{M}_{1}, \mathbf{M}_{2}\right)=\left\|\log \left(\mathbf{M}_{1}^{-1} \mathbf{M}_{2}\right)\right\|$. Thus, the intrinsic mean of a set of m-rep models $\mathbf{M}_{1}, \ldots, \mathbf{M}_{N}$ is the minimizer of the sum-of-squared geodesic distances: $\mu=\underset{\mathbf{M} \in M^{n}}{\arg \min } \sum_{i=1}^{n}\left\|\log \left(\mathbf{M}_{i}^{-1} \mathbf{M}\right)\right\|^{2}$

Principal components of Gaussian data in $\mathbb{R}^{n}$ are defined as the projection onto the linear subspace through the mean spanned by the eigenvectors of the covariance matrix. If we consider a general manifold, the counterpart of a line is a geodesic curve.

As shown in [4], the covariance structure of a Gaussian distribution on $M^{n}$ may be approximated by a covariance matrix $\Sigma$ in the Lie algebra $\mathfrak{m}^{n}$. The eigenvectors of this covariance matrix correspond via the exponential map to geodesics on $M^{n}$, called principal geodesics.

Algorithms for computing the m-rep mean and the principal geodesic analysis on a population of m-rep figures are given in [4].

Analogous to linear PCA models, we may choose a subset of the principal directions $\mathbf{u}^{(k)} \in \mathfrak{m}^{n}$ with corresponding variations $\lambda_{k}$ that is sufficient to describe the variability of the m-rep shape space. New m-rep models may be generated within this subspace of typical objects. Given a set of coefficients $\left\{\alpha_{1}, \ldots, \alpha_{l}\right\}$, we generate a new m-rep model by $\mathbf{M}=\mu \exp \left(\sum_{k=1}^{l} \alpha_{k} \mathbf{u}^{(k)}\right)$, where $\alpha_{k}$ is chosen to be within $\left[-3 \sqrt{\lambda_{k}}, 3 \sqrt{\lambda_{k}}\right]$.

\section{$5 \quad$ Shape Model Bootstrapping}

The segmentation program Pablo provides a user interface that allows construction of m-rep models and optimization of the parameters for fitting to a specific training case 10]. For this work, a batch version of Pablo was developed.

The shape model bootstrapping method is now strikingly simple. From a fiducial starting model, the batch fitting process is used to give rough representations of each shape. The PGA then generates the mean model and corresponding principal geodesics from the 46 fitted prostate models. This mean model is then used to fit the shapes using batch Pablo where the principal geodesics are now used during the figural stage. This bootstrapping procedure is iterated. 

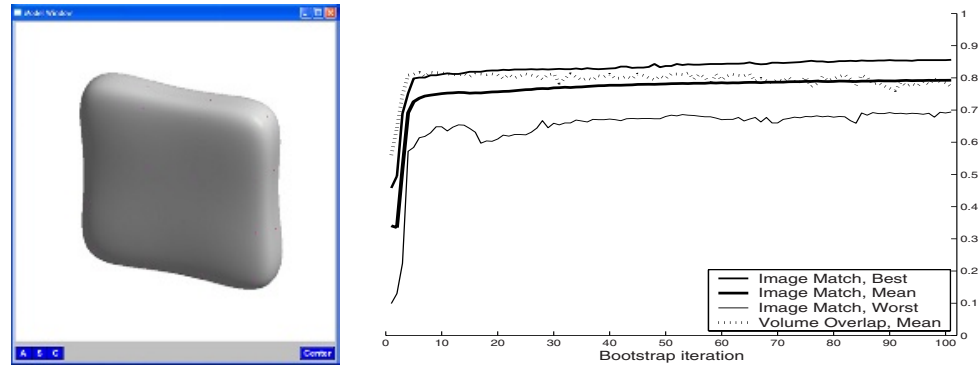

Fig. 2. The Generic model and the resulting bootstrap image match evolution. Image match is as defined in section 3.1 and DICE volume overlap is used (the volume of the intersection between model and manual segmentation divided by their mean volumes).

The idea is that the new mean of the fitted models is a better prototype than the initial model and as the bootstrap iterations progress the generated mean model converges to a good prototype.

\subsection{Bootstrapping from the Generic Model}

The Generic is the default 4x4 m-rep that Pablo generates as a starting model for building handcrafted models. The choice of the specific $4 \mathrm{x} 4$ grid of medial atom reflects a choice of sampling resolution combined with the intention of starting the bootstrap from a neutral, non-committed model. Figure 2 shows the starting model and the progression during bootstrap iterations.

\subsection{Convergence}

In this work, we address the question of convergence pragmatically. The image match and volume overlap values above appear to be converging. Formal proof of the necessary requirements (in terms of starting model and parameter choices) for convergence of the PGA mean and modes is left for future work.

Visual inspection of the mean models after up to 200 bootstrap iterations indicates that the mean is actually converging. Without formal convergence criteria, we use the heuristic approach of running the bootstrap until the image match values cease to improve significantly.

The geometrical regularization introduced in equation 1 is essential to achieving convergence. Without regularization the boundary converges but the medial grid becomes arbitrarily distorted and thereby ruins correspondence - see figure 3 The grid and dist terms keeps the sheet regular and evenly spaced, implode prevents the atoms from collapsing, and swirl keeps the boundary points in proximity to the related atoms. The constrained evolution that is achieved through the geometrical regularization ensures nice correspondence properties through a regular coordinate system on the boundary as well as inside and outside the resulting shape model. 

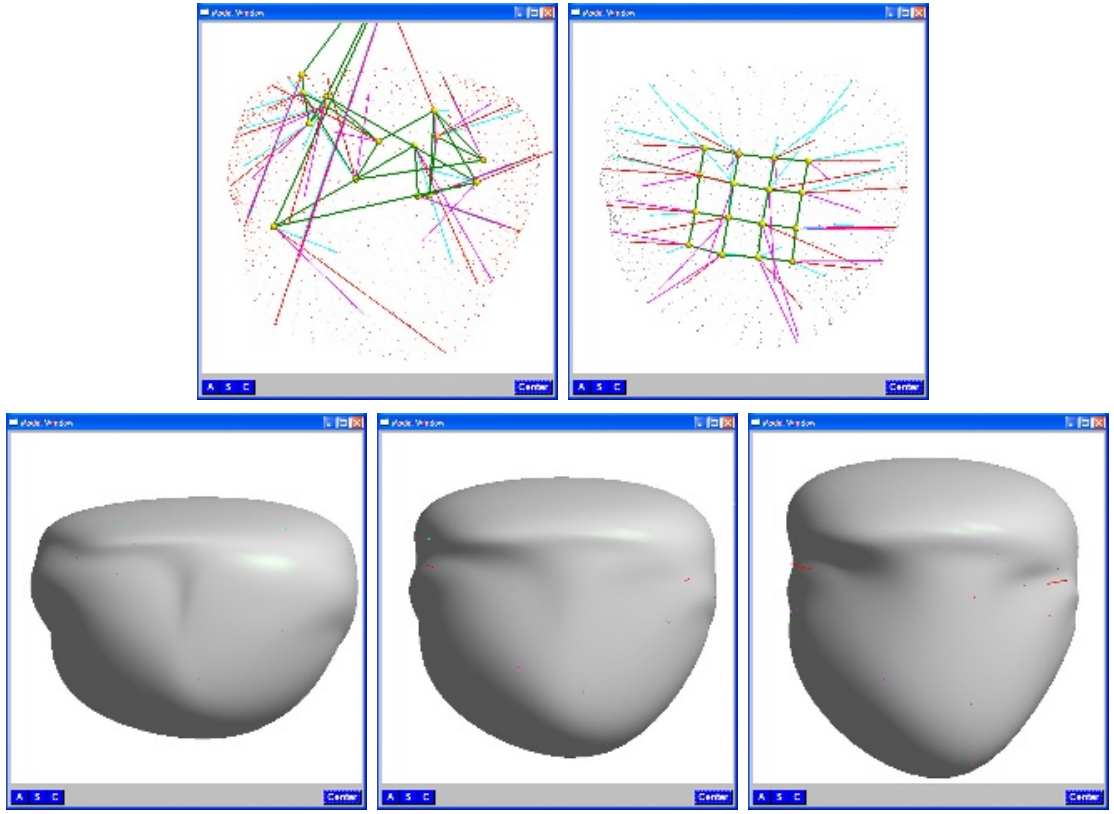

Fig. 3. Top: Comparing medial grid after 100 iterations without and with geometrical regularization $\left(\alpha=0.25, \gamma_{\text {dist }}=0.02, \gamma_{\text {implode }}=0.0002, \gamma_{\text {grid }}=0.002, \gamma_{\text {swirl }}=0.03\right)$. Bottom: Resulting Prostate Mean Model with \pm 1 standard deviation of the first principal geodesic mode of variation - a Laurel/Hardy effect. All seen in anterior-posterior view.

\subsection{Resulting Prostate Shape Model}

The resulting Prostate mean shape model is compared to the result of running the bootstrap without geometrical penalty in figure 3. Without geometrical restrictions, the small deformations in each bootstrap iteration build up and distort the model arbitrarily.

The 10 modes of variation include $98 \%$ of the variation in the training collection. This ensures little need for atom optimization in the segmentation process which speeds up the segmentation method considerably. The automatic fitting achieves image match in the range $0.73-0.87$ with mean 0.82 and a mean DICE volume overlap of 0.80 . We consider this satisfying given the large variation in shape and the non-smooth boundary profiles in the binary segmentations in the training collection (see figure 1).

The shape model is here evaluated on binary images. In the full m-rep segmentation framework, the shape model is combined with profile models for the local boundary (as done in [14 that uses an experimental version of the PGA bootstrap method) instead of just using the Gaussian derivative profile.

Apart from being directly applicable for segmentation, the shape model and the condensed PGA parameterization is applicable for shape classification. 


\section{Conclusion}

We present a novel shape model construction method using a medial shape representation. The method is essentially automatic based on an iterative bootstrap method that alternates between shape representation optimization and principal geodesic analysis of shape mean and variations. The method constructs an m-rep shape model consisting of a mean and corresponding main modes of variation. The non-automatic step is the choice of initial model — we have chosen a generic 4x4 atom grid that is a suitable compromise between compactness and accuracy.

The method is evaluated on a training collection of 46 manually segmented prostates. The resulting model is quantitatively and qualitatively satisfying.

Future work is centered on evaluating our method against the MDL approach that generate an ASM [6]. Central points to evaluate are compactness, correspondence, and legality (how likely are illegal models). Furthermore, modelling of kidneys, hearts and other anatomical structures is to come. Also, ongoing work explores representing medical atoms as a Riemannian symmetric space rather than a Lie group. We have extended the theory of PGA to this space, and future work will involve building the prostate shape model under this new framework.

Acknowledgement. We sincerely thank Per Halverson at Western Wake Radiology (Cary, NC, USA) for supplying pelvis scans for the collection.

\section{References}

1. Cootes, T., Taylor, C., Cooper, D., Graham, J.: Active shape models: Their training and application. CVIU (1995) 38-59

2. Cremers, D., Kohlberger, T., Schnörr, C.: Nonlinear shape statistics in mumfordshah based segmentation. In: 7th European Conference on Computer Vision. Volume 2351 of Springer LNCS. (2002)

3. Pizer, S., Fritsch, D., Yushkevich, P., Johnson, V., Chaney, E.: Segmentation, registration, and measurement of shape variation via image object shape. IEEE Transactions on Medical Imaging 18 (1996)

4. Fletcher, P.T., Joshi, S., Lu, C., Pizer, S.M.: Gaussian distributions on Lie groups and their application to statistical shape analysis. To appear Information Processing in Medical Imaging (2003)

5. Fletcher, P.T., Lu, C., Joshi, S.: Statistics of shape via principal geodesic analysis on Lie groups. To appear Computer Vision and Pattern Recognition (2003)

6. Davies, R., Twining, C., Cootes, T., Waterton, J., Taylor, C.: A minimum description length approach to statistical shape modeling. IEEE Transactions on Medical Imaging 21 (2002)

7. Styner, M., Gerig, G.: Medial models incorporating object variability for 3d shape analysis. In: Proc. of Information Processing in Medical Imaging. (2001)

8. Guimond, A., Meunier, J., Thirion, J.P.: Average brain models: A convergence study. Technical Report 3731, INRIA (1999)

9. Tracton, G., Chaney, E., Rosenman, J., Pizer, S.: Mask: combining 2d and 3d segmentation methods to enhance functionality. In: Proceedings of Mathematical Methods in Medical Imaging III. Volume SPIE Vol 2299. (1994) 
10. Pizer, Chen, Fletcher, Fridman, Fritsch, Gash, Glotzer, Jiroutek, Joshi, Muller, Thall, Tracton, Yushkevich, Chaney: Deformable m-reps for 3d medical image segmentation. IJCV (2003)

11. Joshi, S., Pizer, S., Fletcher, P.T., Yushkevich, P., Thall, A., Marron, J.S.: Multiscale deformable model segmentation and statistical shape analysis using medial descriptions. Transactions on Medical Imaging 21 (2002)

12. Blum, H., Nagel, R.: Shape description using weighted symmetric axis features. Pattern Recognition 10 (1978) 167-180

13. Thall, A.: Fast $C^{2}$ interpolating subdivision surfaces using iterative inversion of stationary subdivision rules. Technical report, UNC (2002) http://midag.cs.unc.edu/pubs/papers/Thall_TR02-001.pdf.

14. Rao, M.: Analysis of a locally varying intensity template for segmentation of kidneys in ct images. Master's thesis, UNC, Chapel Hill (2003) 